\title{
Image quality and radiation dose comparison of prospectively triggered low-dose CCTA: 128-slice dual-source high-pitch spiral versus 64-slice single-source sequential acquisition
}

\author{
Ghadri, J R ; Küest, S M ; Goetti, R ; Fiechter, M ; Pazhenkottil, A P ; Nkoulou, R N ; Kuhn, F P ;
} Pietsch, C ; von Schulthess, P ; Gaemperli, O ; Templin, C ; Kaufmann, P A

\begin{abstract}
Currently 64-multislice computed tomography (MSCT) scanners are the most widely used devices allowing low radiation dose coronary CT angiography (CCTA) with prospective ECG triggering. Latest 128-slice dual-source CT (DSCT) scanners offer prospective high-pitch spiral acquisition covering the heart during one single beat. We compared radiation dose and image quality from prospective 64MSCT versus high-pitch spiral 128-slice DSCT scanning, as such data is lacking. CCTA of 50 consecutive patients undergoing 128 -DSCT $(2 \times 64 \times 0.6 \mathrm{~mm}$ collimation, 0.28 s rotation time, 3.4 pitch, 100-120 $\mathrm{kV}$ tube voltage and $320 \mathrm{mAs}$ tube current-time product) were compared to CCTA of 50 heart rate $(\mathrm{HR})$ and BMI matched patients undergoing 64 -MSCT $(64 \times 0.625 \mathrm{~mm}$ collimation, $0.35 \mathrm{~s}$ rotation time, $100-120 \mathrm{kV}$ tube voltage and 400-650 mA tube current). Image quality was rated on a 4-point scale by two independent cardiac imaging physicians $(1=$ excellent to $4=$ non-diagnostic). Of 710 coronary segments assessed on 128-DSCT, 216 (30.4\%) achieved an image quality score 1 excellent, $400(56.3 \%)$ score $2,76(10.7 \%)$ score 3 and $18(2.6 \%)$ score 4 (non-diagnostic). Of 737 coronary segments evaluated on 64-MSCT 271 (36.8\%) had an image quality score of 1, $327(44.4 \%) 2,110$ (14.9\%) score 3, and 29 $(3.9 \%)$ segments score 4 . Average image quality score for both scanners was similar $(\mathrm{P}=0.641)$. The mean heart rate during scanning was $58.7 \pm 5.6 \mathrm{bpm}$ on 128-DSCT and $59.0 \pm 5.6 \mathrm{bpm}$ on 64-MSCT, respectively. Mean effective radiation dose was $1.0 \pm 0.2 \mathrm{mSv}$ for 128 -DSCT and $1.7 \pm 0.6 \mathrm{mSv}$ for 64-MSCT $(\mathrm{P}<0.001)$. 128-DSCT with high-pitch spiral mode allows CCTA acquisition with reduced radiation dose at maintained image quality compared to 64-MSCT.
\end{abstract}

DOI: https://doi.org/10.1007/s10554-011-9921-3

Posted at the Zurich Open Repository and Archive, University of Zurich

ZORA URL: https://doi.org/10.5167/uzh-51125

Journal Article

Published Version

Originally published at:

Ghadri, J R; Küest, S M; Goetti, R; Fiechter, M; Pazhenkottil, A P; Nkoulou, R N; Kuhn, F P; Pietsch, C; von Schulthess, P; Gaemperli, O; Templin, C; Kaufmann, P A (2012). Image quality and radiation dose comparison of prospectively triggered low-dose CCTA: 128-slice dual-source high-pitch spiral versus 64-slice single-source sequential acquisition. International Journal of Cardiovascular Imaging, 28(5):12171225 .

DOI: https://doi.org/10.1007/s10554-011-9921-3 


\title{
Image quality and radiation dose comparison of prospectively triggered low-dose CCTA: 128-slice dual-source high-pitch spiral versus 64-slice single-source sequential acquisition
}

\author{
Jelena R. Ghadri • Silke M. Küest • Robert Goetti • Michael Fiechter • \\ Aju P. Pazhenkottil • Rene N. Nkoulou • Felix P. Kuhn • Carsten Pietsch • \\ Patrick von Schulthess · Oliver Gaemperli · Christian Templin · Philipp A. Kaufmann
}

Received: 7 June 2011/Accepted: 4 July 2011/Published online: 10 July 2011

(C) Springer Science+Business Media, B.V. 2011

\begin{abstract}
Currently 64-multislice computed tomography (MSCT) scanners are the most widely used devices allowing low radiation dose coronary CT angiography (CCTA) with prospective ECG triggering. Latest 128-slice dual-source CT (DSCT) scanners offer prospective high-pitch spiral acquisition covering the heart during one single beat. We compared radiation dose and image quality from prospective 64-MSCT versus high-pitch spiral 128-slice DSCT scanning, as such data is lacking. CCTA of 50 consecutive patients undergoing 128-DSCT $(2 \times$ $64 \times 0.6 \mathrm{~mm}$ collimation, $0.28 \mathrm{~s}$ rotation time, 3.4 pitch, 100-120 kV tube voltage and $320 \mathrm{mAs}$ tube current-time product) were compared to CCTA of 50 heart rate (HR) and BMI matched patients undergoing $64-\mathrm{MSCT}(64 \times 0.625 \mathrm{~mm}$ collimation, $0.35 \mathrm{~s}$ rotation time, $100-120 \mathrm{kV}$ tube voltage and $400-650 \mathrm{~mA}$
\end{abstract}

Jelena R. Ghadri and Silke M. Küest equally contributed to this work.

J. R. Ghadri · S. M. Küest · R. Goetti · M. Fiechter ·

A. P. Pazhenkottil · R. N. Nkoulou · F. P. Kuhn .

C. Pietsch · P. von Schulthess · O. Gaemperli .

C. Templin · P. A. Kaufmann ( $\square)$

Department of Radiology, Cardiac Imaging, University

Hospital Zurich, Ramistrasse 100, NUK C 42,

8091 Zurich, Switzerland

e-mail:pak@usz.ch

M. Fiechter · P. A. Kaufmann

Zurich Centre for Integrative Human Physiology (ZIHP),

University of Zurich, Zurich, Switzerland tube current). Image quality was rated on a 4-point scale by two independent cardiac imaging physicians ( 1 = excellent to $4=$ non-diagnostic). Of 710 coronary segments assessed on 128-DSCT, 216 (30.4\%) achieved an image quality score 1 excellent, 400 (56.3\%) score 2, $76(10.7 \%)$ score 3 and 18 (2.6\%) score 4 (non-diagnostic). Of 737 coronary segments evaluated on 64-MSCT 271 (36.8\%) had an image quality score of 1, $327(44.4 \%) 2,110$ (14.9\%) score 3 , and 29 (3.9\%) segments score 4. Average image quality score for both scanners was similar $(P=$ $0.641)$. The mean heart rate during scanning was $58.7 \pm 5.6 \mathrm{bpm}$ on 128-DSCT and $59.0 \pm 5.6 \mathrm{bpm}$ on 64-MSCT, respectively. Mean effective radiation dose was $1.0 \pm 0.2 \mathrm{mSv}$ for 128-DSCT and $1.7 \pm$ $0.6 \mathrm{mSv}$ for 64-MSCT $(P<0.001)$. 128-DSCT with high-pitch spiral mode allows CCTA acquisition with reduced radiation dose at maintained image quality compared to 64-MSCT.

Keywords Coronary artery disease . 64-Slice MSCT · 128-Slice DSCT · Image quality • Radiation dose

\section{Introduction}

The clinical use of coronary computed tomography angiography (CCTA) in patients with low to intermediate risk for coronary artery disease (CAD) has substantially increased over the past decade. This is 
not only due to the widespread availability and accessibility of this technique, but it has been substantially facilitated by the introduction of prospective ECG-triggering. This has resulted in a massive radiation dose reduction from over $30 \mathrm{mSv}$ [1] to around $2 \mathrm{mSv}$ and therefore referred to as lowdose CT [2-4] by confining the CT-scan to the smallest possible window at only one distinct middiastolic phase of the heart cycle while the X-ray tube is turned off during the rest of the cycle. The beauty of this prospectively gated sequential protocol originally reported for 64-slice CT (MSCT) [2] is the fact that it can be applied to different types of scanners from all vendors including latest generation technology such as 128- or 320-slice CT [5-7].

Recently, the prospectively triggered high-pitch spiral mode has been described as an alternative acquisition protocol for low-dose CCTA) [8-10]. However, in order to scan the entire heart in a spiral mode within one single cardiac cycle, a very highpitch of $>3$ is necessary even when using a dualsource CT (DSCT) scanner equipped with two 128 -slice acquisition detectors. In patients with very low heart rates this highly sophisticated technique can reduce radiation dose for CCTA to $1 \mathrm{mSv}$ or below which is lower than the values reported for sequential scanning with the most widely used 64-slice scanner generation. However, as most centres are not equipped with CT devices from different technology generations and across different vendors, direct comparison is lacking.

Therefore, the aim of the present study was to prospectively compare CCTA image quality and radiation dose from 128-DSCT with prospective high-pitch spiral mode versus 64-MSCT with prospectively triggered sequential scanning.

\section{Materials and methods}

\section{Study population}

We included 50 consecutive patients undergoing CCTA by 128-DSCT scanning and 50 patients matched for heart rate and BMI who were scanned with 64-MSCT to exclude possible CAD or to evaluate known or suspected CAD. Patients were included if they had signed informed consent authorizing their records to be included in our CCTA research registry.
Exclusion criteria were: allergy to contrast agent, nephropathy (creatinine level $>150 \mu \mathrm{mol} / \mathrm{l}, 1.7 \mathrm{mg} /$ $\mathrm{dl}$ ), nonsinus rhythm, heart rate $>70 \mathrm{bpm}$ after intravenous beta-blocker administration, hemodynamic instability or pregnancy.

Before the study, a detailed interview was conducted to collect data on symptoms, previous cardiac events, and cardiovascular risk factors and collected in our CCTA registry.

\section{CT acquisition and image reconstruction}

Before CT scanning all patients received a single sublingual dose of isosorbide dinitrate $2.5 \mathrm{mg}$ (Isoket; Schwarz Pharma, Monheim, Germany) $2 \mathrm{~min}$ before scanning [4]. Intravenous metoprolol (Beloc, AstraZeneca, London, UK) was administered to achieve a heart rate less than $70 \mathrm{bpm}$, as previously reported [7].

CCTA scanning was performed on a secondgeneration DSCT acquiring 128-slices by use of a z-flying focal spot (128-DSCT, Somatom Definition Flash, Siemens Healthcare, Forchheim, Germany) and a 64-slice MSCT (LightSpeed VCT XT, GE Healthcare, Milwaukee, USA). All patients were instructed about breath holding and the importance of immobility during scanning. All scans on both scanners were performed in cranio-caudal direction with prospective electrocardiogram ECG-triggering. Scanning parameters for 128-DSCT were as follows: center of data acquisition starting at $60 \%$ of the RR interval, slice collimation $2 \times 64 \times 0.6 \mathrm{~mm}$ by means of a z-flying focal spot, gantry rotation time $280 \mathrm{~ms}$, pitch 3.4 , tube voltage 100 or $120 \mathrm{kV}$ $\left(\right.$ BMI $<25 \mathrm{~kg} / \mathrm{m}^{2}$ or BMI $>25 \mathrm{~kg} / \mathrm{m}^{2}$ ), tube current-time product $320 \mathrm{mAs}$. A fixed intravenous bolus of $80 \mathrm{ml}$ iopromide (Ultravist $300,370 \mathrm{mg} / \mathrm{ml}$, Bayer Schering Pharma, Berlin, Germany) was administered at a flow rate of $5 \mathrm{ml} / \mathrm{s}$ followed by $60 \mathrm{ml}$ saline solution [11]. Contrast injection was timed by bolus tracking in a sampling region of interest the ascending aorta with the scan beginning $10 \mathrm{~s}$ after exceeding a threshold of 100 Hounsfield units (HU). Images were reconstructed with a slice thickness of $0.8 \mathrm{~mm}$ and increment of $0.4 \mathrm{~mm}$ using a medium smooth kernel designed for cardiac imaging (B26f). All images were anonymised and transferred to an external workstation (AW 4.4, GE Healthcare) for evaluation. 
For 64-MSCT a sequential scanning mode with the center of acquisition at $75 \%$ of the RR-interval without padding was applied with the following scanning parameters [12]: slice collimation $64 \times 0.625 \mathrm{~mm}$; z-coverage, $40 \mathrm{~mm}$ with an increment of $35 \mathrm{~mm}$, gantry rotation time, $350 \mathrm{~ms}$; tube voltage and tube current adapted to BMI $(26.3 \pm 3.7)$. Images were reconstructed with a slice thickness of $0.625 \mathrm{~mm}$, using a medium-soft tissue convolution kernel (standard). We used a BMI-adapted contrast material protocol, which has been previously established $[10,13,14]$.

Of note, according to our standard routine protocol, no additional coronary calcium scanning was performed.

Image quality of coronary artery segments

According to the 16-segment model of the American Heart Association [15]. All coronary segments were assigned to one of the main coronary arteries as follows: right coronary artery (RCA) segments 1-4, left main artery (LMA) and the left anterior ascending artery (LAD) segments 5-10, and the left circumflex artery (LCX) segments 11-15, intermediate artery segment 16 , if present. The image quality was visually assessed by two independent readers for each segment on a 4-point scale using axial source images and multiplanar reformations, whereby score $1=$ excellent (no motion artefacts); score $2=$ good (minor artefacts); score $3=$ adequate (moderate artefacts); score $4=$ non-diagnostic, as previously reported [3]. For any disagreement in data evaluation between the two readers, consensus agreement was achieved.

Image noise and contrast-to-noise ratio (CNR) were determined by one observer not involved in the image quality score rating. The vessel contrast was measured as the difference in mean attenuation in $\mathrm{HU}$ in the contrast enhanced vessel lumen and the mean attenuation in $\mathrm{HU}$ in the adjacent perivascular tissue. Attenuation was measured in round-shaped regions of interest (ROI) in the proximal LMA and RCA (measurements 1 and 2). Image noise was defined as the SD of the attenuation value in a ROI placed in the ascending aorta. All ROIs were drawn as large as possible, carefully avoiding the vessel wall and blooming halo of calcifications. CNR was calculated as the ratio of the mean of measurements 1 and 2, and image noise as previously described [16, 17].
Radiation dose

Values for effective radiation dose were calculated by multiplying the dose length product (DLP) with a conversion factor for the chest $(k=0.014 \mathrm{mSv} /$ mGyxcm) as previously suggested [18] and adopted in large trials[1].

Statistical analysis

Statistical analysis was performed using the SPSS software (19.0, SPSS inc., Chicago IL, USA). Quantitative variables were expressed as mean \pm standard deviation (SD) or median (range) as appropriate, and categorical variables as frequencies, or percentages.

The Mann-Whitney $U$ test was performed to analyse the differences between the two groups regarding image quality, radiation dose, image noise and contrast-to-noise ratio. The student's $t$ test or Mann-Whitney U test was used to compare the means of the continuous variables (demographic data) and contingency tables (risk factors, referral indications, CAD data. Image artefact per patient) were analyzed using chi-square test.

Interobserver agreement was evaluated by Cohen's Kappa coefficient. A $k$ value of greater of 0.81 was defined as excellent, $0.61-0.80$ was considered as good, values of $0.41-0.60$ as moderate and values below 0.20 as poor agreement.

A two-tailed $P$ value $<0.05$ was considered statistically significant. Comparison of patient characteristics was evaluated with Chi-squared test for unnormal distribution. The impact on mean heart rate on image quality was assessed by Spearman's rankorder. Correlation coefficients were calculated to compare HR with the mean image quality scores of all coronary segments on a per patient basis.

\section{Results}

Study population

All 100 patients underwent successful scanning with either high-pitch acquisition on 128-DSCT or sequential scanning on 64-MSCT. The mean age of the 128-DSCT group was $57.5 \pm 13.1$ years and $59.9 \pm$ 12.2 for the $64-\mathrm{MSCT}$ group $(P=0.46)$. Patients were adequately matched regarding heart rate $(P=0.69)$ 
and BMI $(P=0.81)$ and there were no significant differences between the two groups regarding cardiovascular risk factors. The patient baseline characteristics, referral indications and previous state of CAD are given in Table 1.

Subjective image quality

Image quality was assessed for all 1,447 coronary segments from 128-DSCT $(n=710)$ and for 64-MSCT ( $n=737$ ) (Table 2). The total number of segments is not equal due to anatomic variations with not all segments being present in all patients. Interobserver agreement for rating image quality

Table 1 Baseline characteristics

\begin{tabular}{|c|c|c|c|}
\hline Demographics & $\begin{array}{l}128-\mathrm{DSCT} \\
n=50\end{array}$ & $\begin{array}{l}\text { 64-MSCT } \\
n=50\end{array}$ & $P$ \\
\hline Male & $35(70 \%)$ & $33(66 \%)$ & 0.409 \\
\hline Age (years) & $57.5 \pm 13.1$ & $59.9 \pm 12.2$ & 0.469 \\
\hline Weight (kg) & $77.4 \pm 11.9$ & $78.6 \pm 14.5$ & 0.735 \\
\hline Height (cm) & $171.4 \pm 9.5$ & $172.5 \pm 9.1$ & 0.756 \\
\hline BMI $\left(\mathrm{kg} / \mathrm{m}^{2}\right)$ & $26.4 \pm 3.4$ & $26.3 \pm 3.7$ & 0.817 \\
\hline $\begin{array}{l}\text { Heart rate during scan } \\
\text { (bpm) }\end{array}$ & $58.7 \pm 5.6$ & $59.0 \pm 5.6$ & 0.694 \\
\hline \multicolumn{4}{|l|}{ Risk factors } \\
\hline Hypertension & $27(54 \%)$ & $31(62 \%)$ & 0.417 \\
\hline Dyslipidaemia & $25(50 \%)$ & $24(48 \%)$ & 0.841 \\
\hline Diabetes mellitus & $4(8 \%)$ & $5(10 \%)$ & 0.726 \\
\hline Smoking & $14(28 \%)$ & $22(44 \%)$ & 0.095 \\
\hline Family history & $19(38 \%)$ & $19(38 \%)$ & 1.000 \\
\hline \multicolumn{4}{|l|}{ Referral indication } \\
\hline Chest pain & $11(22 \%)$ & $10(20 \%)$ & 0.814 \\
\hline Atypical chest pain & $5(10 \%)$ & $17(34 \%)$ & 0.004 \\
\hline None & $11(22 \%)$ & $11(22 \%)$ & 1.000 \\
\hline Dyspnoea & $8(16 \%)$ & $2(4 \%)$ & 0.045 \\
\hline $\begin{array}{c}\text { Pre-operative } \\
\text { assessment }\end{array}$ & $8(16 \%)$ & $4(8 \%)$ & 0.218 \\
\hline Bicycle test pathologic & $6(12 \%)$ & $4(8 \%)$ & 0.444 \\
\hline Check-up & $1(2 \%)$ & $2(4 \%)$ & 0.557 \\
\hline \multicolumn{4}{|c|}{ Coronary artery disease (CAD) } \\
\hline Known CAD & $7(14 \%)$ & $11(22 \%)$ & 0.297 \\
\hline Previous bypass & $4(8 \%)$ & $4(8 \%)$ & 1.000 \\
\hline Previous stenting & $3(6 \%)$ & $5(10 \%)$ & 0.461 \\
\hline $\begin{array}{l}\text { Prior myocardial } \\
\text { infarction }\end{array}$ & $1(2 \%)$ & $6(12 \%)$ & 0.050 \\
\hline
\end{tabular}

$B M I$ body mass index, $C A D$ coronary artery disease was good for high-pitch spiral mode (128-DSCT; $k=0.66)$ and for sequential scanning (64-MSCT; $k=0.61)$.

Among the 128-DSCT scans 692 (97.5\%) segments yielded diagnostic image quality (score 1-3), which was excellent in 216 (30.4\%), good in 400 $(56.3 \%)$ and adequate in $76(10.7 \%)$ coronary segments (Table 3). Similarly among the 64-MSCT scans $708(96.1 \%)$ coronary segments revealed diagnostic image quality (score 1-3), which was excellent in $271(36.8 \%)$, good in $327(44.4 \%)$ and adequate in $110(14.9 \%)$ coronary segments (Table 3). Figure 1 demonstrates the frequency of image quality for overall coronary segments for 128-DSCT and 64-MSCT.

Causes for poor image quality were the presence of motion or step-artefacts. None of the patients scanned with 128-DSCT revealed step-artefacts, however motion artefacts occurred in 14 patients

Table 2 Mean image quality score

\begin{tabular}{|c|c|c|}
\hline & 128-DSCT & 64-MSCT \\
\hline \multicolumn{3}{|l|}{ Per segment } \\
\hline Segment 1 & 1.65 & 1.60 \\
\hline Segment 2 & 2.12 & 2.44 \\
\hline Segment 3 & 2.58 & 2.57 \\
\hline Segment 4 & 1.90 & 1.71 \\
\hline Segment 5 & 1.37 & 1.24 \\
\hline Segment 6 & 1.40 & 1.38 \\
\hline Segment 7 & 1.76 & 1.86 \\
\hline Segment 8 & 2.18 & 2.32 \\
\hline Segment 9 & 1.90 & 1.78 \\
\hline Segment 10 & 1.93 & 2.00 \\
\hline Segment 11 & 1.62 & 1.35 \\
\hline Segment 12 & 1.74 & 1.77 \\
\hline Segment 13 & 1.74 & 1.72 \\
\hline Segment 14 & 2.00 & 2.09 \\
\hline Segment 15 & 2.00 & 2.44 \\
\hline Segment 16 & 1.87 & 1.67 \\
\hline \multicolumn{3}{|l|}{ Per vessel } \\
\hline $\mathrm{RCA}$ & 2.06 & 2.09 \\
\hline LMA/LAD & 1.75 & 1.76 \\
\hline LCX & 1.80 & 1.80 \\
\hline Per patient & 1.85 & 1.86 \\
\hline
\end{tabular}

$R C A$ right coronary artery, $L M A$ left main artery, $L A D$ left anterior descending artery, $L C X$ left circumflex artery 
Table 3 Image Quality total (128-DSCT $n=710$; 64-MSCT $n=737$; total segments $n=1447$ )

\begin{tabular}{lcll}
\hline Total segments & $\begin{array}{l}\text { 128-DSCT } \\
710\end{array}$ & $\begin{array}{l}\text { 64-MSCT } \\
737\end{array}$ & $P$ \\
\hline Score 1 & $216(30.4 \%)$ & $271(36.8 \%)$ & 0.299 \\
Score 2 & $400(56.3 \%)$ & $327(44.4 \%)$ & 0.03 \\
Score 3 & $76(10.7 \%)$ & $110(14.9 \%)$ & 0.256 \\
Score 4 & $18(2.6 \%)$ & $29(3.9 \%)$ & 0.984 \\
\hline
\end{tabular}

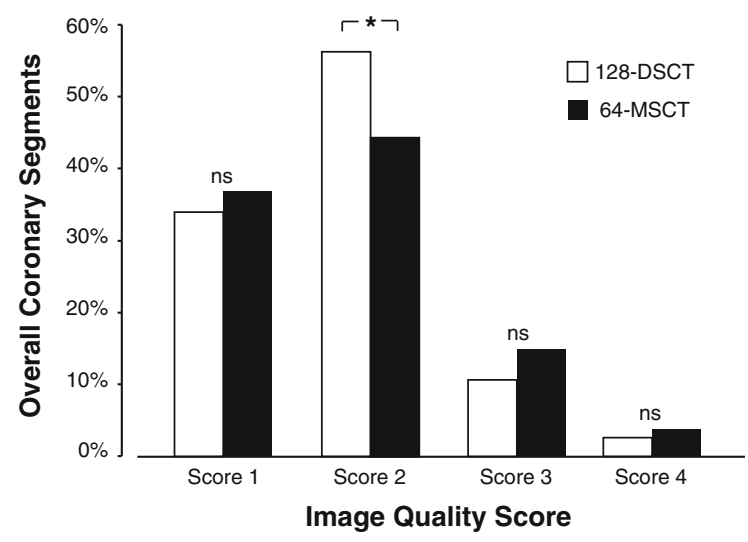

Fig. 1 Frequency of image quality for overall coronary segments for 128-DSCT (white bars) and 64-MSCT (black bars $),\left({ }^{*} P=0.03 ; n s\right.$ not significant)

Table 4 Image artifact per patient

\begin{tabular}{llll}
\hline & $\begin{array}{l}\text { 128-DSCT } \\
n=50\end{array}$ & $\begin{array}{l}\text { 64-MSCT } \\
n=50\end{array}$ & $P$ \\
\hline Step artefact & None & $9(18 \%)$ & 0.001 \\
Motion artefact & $14(28 \%)$ & $10(20 \%)$ & 0.349 \\
\hline
\end{tabular}

(28\%). In the 64-MSCT group nine patients (18\%) revealed step artefacts, and $10(20 \%)$ motion artefacts (Table 4).

There was no significant difference $(P=0.641)$ in image quality between the two scanners with different scanning protocols for any of the coronary segments as well as for the overall image quality.

Impact of heart rate on image quality

The average heart during scanning was $58.7 \pm$ $5.6 \mathrm{bpm}$ (range 46-70 bpm) for 128-DSCT and $59.0 \pm 5.6 \mathrm{bpm}$ (range $47-70 \mathrm{bpm}$ ) for $64-\mathrm{MSCT}$.
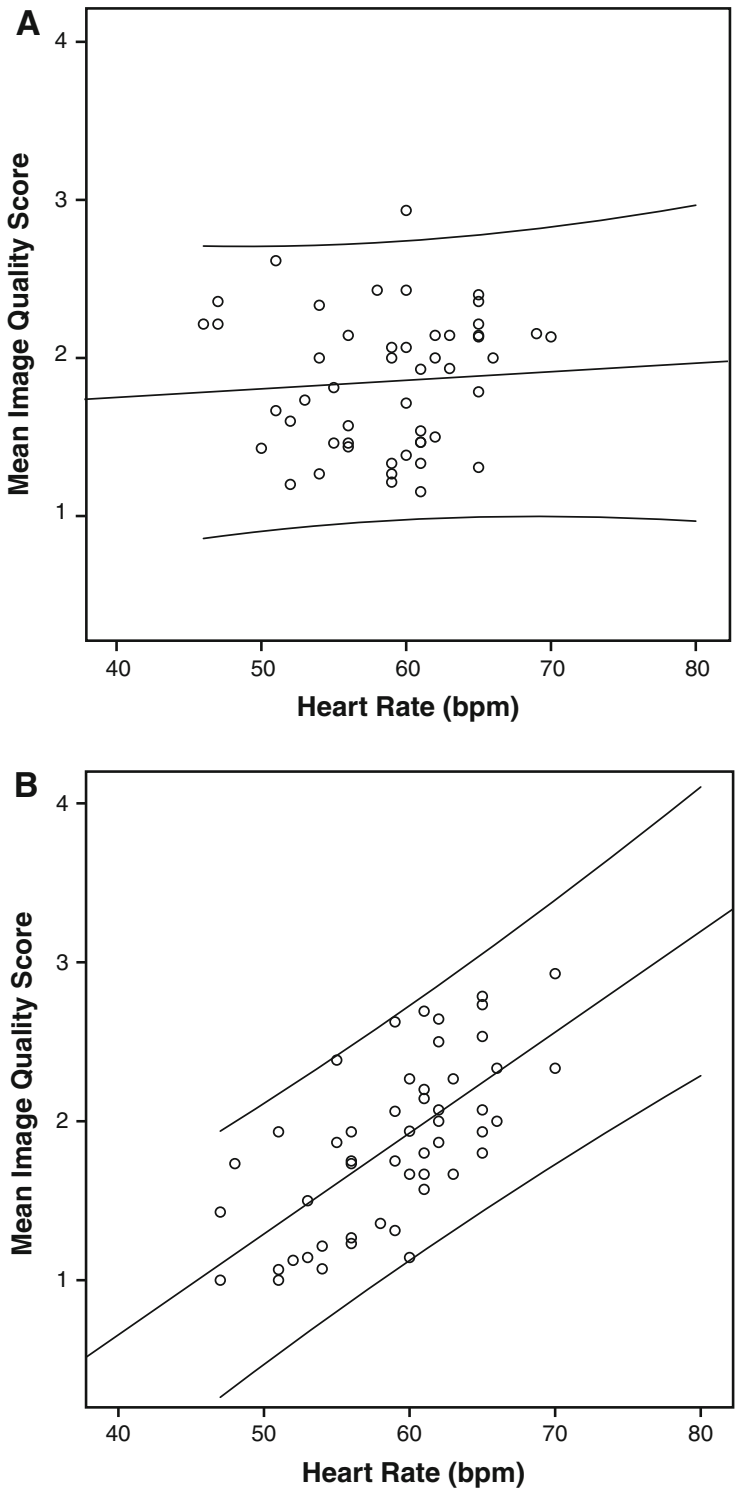

Fig. 2 Linear regression plot of mean image quality scores over all coronary segments per patient ( $y$-axis) against heart rate during CT-scanning ( $x$-axis) in 128-DSCT a and 64-MSCT b. The two outer lines represent $95 \%$ individual prediction interval (Spearman's correlation for 128-DSCT $r=0.11$, $P=0.45$; Spearman's correlation for 64-MSCT $r=0.68$, $P<0.01)$

There was no significant correlation between HR and mean image quality score on per patient analysis ( $r=0.11, P=0.45$ ) for 128 -DSCT, while a significant correlation between heart rate and image quality was found for 64-MSCT scanning $(r=0.68$, $P \leq 0.01)$, Fig. 2a and b. 
Table 5 Radiation dose

\begin{tabular}{lccl}
\hline & 128-DSCT & 64-MSCT & $P$ \\
\hline CTDI (mGy) & $3.3 \pm 0.8$ & $8.2 \pm 2.8$ & $<0.001$ \\
DLP (mGyxcm) & $71.2 \pm 17.7$ & $124.5 \pm 41.7$ & $<0.001$ \\
$\begin{array}{l}\text { Effective radiation } \\
\quad \text { dose (mSv) }\end{array}$ & $1.0 \pm 0.3$ & $1.7 \pm 0.6$ & $<0.001$ \\
Scan length (cm) & $14.4 \pm 2.6$ & $14.9 \pm 2.2$ & 0.306 \\
\hline
\end{tabular}

CTDI Computed tomography dose index, DLP dose length product

Objective image quality

Contrast was significantly different between 128-DSCT and 64 MSCT $(532.7 \pm 122.0$ vs. $502.8 \pm 84.0$; $P=0.014)$. Image noise was not significantly different (128-DSCT: $31.5 \pm 8.3 \mathrm{HU}$; 64-MSCT: $34.6 \pm 8.8$ $\mathrm{HU} ; \mathrm{p}=0.056)$. CNR was $(18.3 \pm 5.5$ vs. $14.6 \pm 4.4$; $P<0.01)$.

Radiation dose

There was a significant difference in the effective radiation dose between the 128-DSCT high-pitch group $(1.0 \pm 0.3 \mathrm{mSv})$ and the 64-MSCT sequential scanning group $(1.7 \pm 0.6 \mathrm{mSv})(P>0.001)$. The average DLP was $71.2 \pm 17.7$ (mGyxcm) on 128-DSCT and $124.5 \pm 41.7$ (mGyxcm) on 64-MSCT, although the scan length did not differ significantly between the two $(14.4 \pm 2.6 \mathrm{~cm}$ vs. $14.9 \pm 2.2 \mathrm{~cm}, P=$ n.s. $)$ (Table 5). The mean tube current-time product/ tube voltage was $383 \pm 43 \mathrm{mAs} / 107 \pm 10 \mathrm{kV}$ for 64-MSCT and $327 \pm 22 \mathrm{mAs} / 101 \pm 5 \mathrm{kV}$ for 128-DSCT.

\section{Discussion}

The recent years have witnessed tremendous technological advances in multislice CT systems enabling high image quality CCTA in clinical practice while constantly reducing radiation exposure for the patient. Since there is no threshold below which radiation exposure can be proven to not be potentially cancerogenous, any radiation dose can be potentially harmful and should therefore be minimized [19].

This is the first study with a cross-vendor comparison of CCTA image quality and radiation dose from 128-DSCT versus 64-MSCT. In fact, much effort has been invested in developing methods to improve image quality and/or reduce radiation dose in CCTA. Unfortunately, most approaches to solve one issue act in opposite direction on the other issue. Our results document that CCTA with 128-DSCT using highpitch scanning allows further substantial reduction in radiation exposure compared to low-dose CCTA with prospective sequential scanning. This was achieved at maintained image quality, which is of great importance in the context mentioned above (Fig. 3). The amount of radiation dose reduction is in line with previous reports of radiation dose saving with new generation scanners $[7,20]$ Recent data have demonstrated the feasibility of prospective ECG-triggering with high-pitch spiral CCTA acquisition with sub-mSv radiation doses [8]. However, none of these studies have compared radiation doses and image quality of CCTA from different systems of different vendors.

In our study we found a difference of the mean effective radiation dose between the two groups of $0.7 \mathrm{mSv}$, which implies a dose reduction of $41.2 \%$ when using the high-pitch 128-DSCT spiral protocol. Although an absolute decrease in radiation dose of $0.7 \mathrm{mSv}$ may appear rather modest with regards to the massive increase in cost from single to dual source scanners, even this radiation dose saving may be worth its cost, as the radiation dose for a CCTA now is as low as a fraction of the annual background exposure of approximately $3.2 \mathrm{mSv}$ by radon [21]. In fact, since there is no threshold below which radiation has been proven to not be cancerogenous, any amount of radiation must be considered potentially harmful. Consequently, any amount of dose reduction should be considered worth the cost.

The observed dose reduction despite the same scan length (which otherwise is a major determinant of the dose-length-product, DLP), may be explained considering various factors. First, tube voltage and tube current settings are not directly comparable between the two protocols used in this study. However, we have used well established protocols specific for high-pitch spiral $[7,20]$ and prospectively triggered sequential scanning [22]. Second, inherent differences between spiral and sequential protocol may have contributed to the dose reduction. For example oversampling is minimized using the high-pitch dualspiral technique, while a certain overlap of the individual blockwise scans that comprise a study is necessary for precise image reconstruction in 
Fig. 3 Prospectively ECGtriggered images on 128-DSCT $(\mathbf{a}-\mathbf{c})$ and 64-MSCT (d-f) of the right coronary artery (RCA) in volume rendered multiplanar reconstruction and curved multiplanar reformation. a-c, shows RCA in a in a 45 year old patient with an average heart rate of $59 / \mathrm{min}$ and a BMI $29.07 \mathrm{~kg} / \mathrm{m}^{2}$. d-f, shows RCA in a 60 year old patient with a heart rate 59/min and a BMI $29.07 \mathrm{~kg} / \mathrm{m}^{2}$, respectively. Image quality was scored with 1 (=excellent) for both scanners
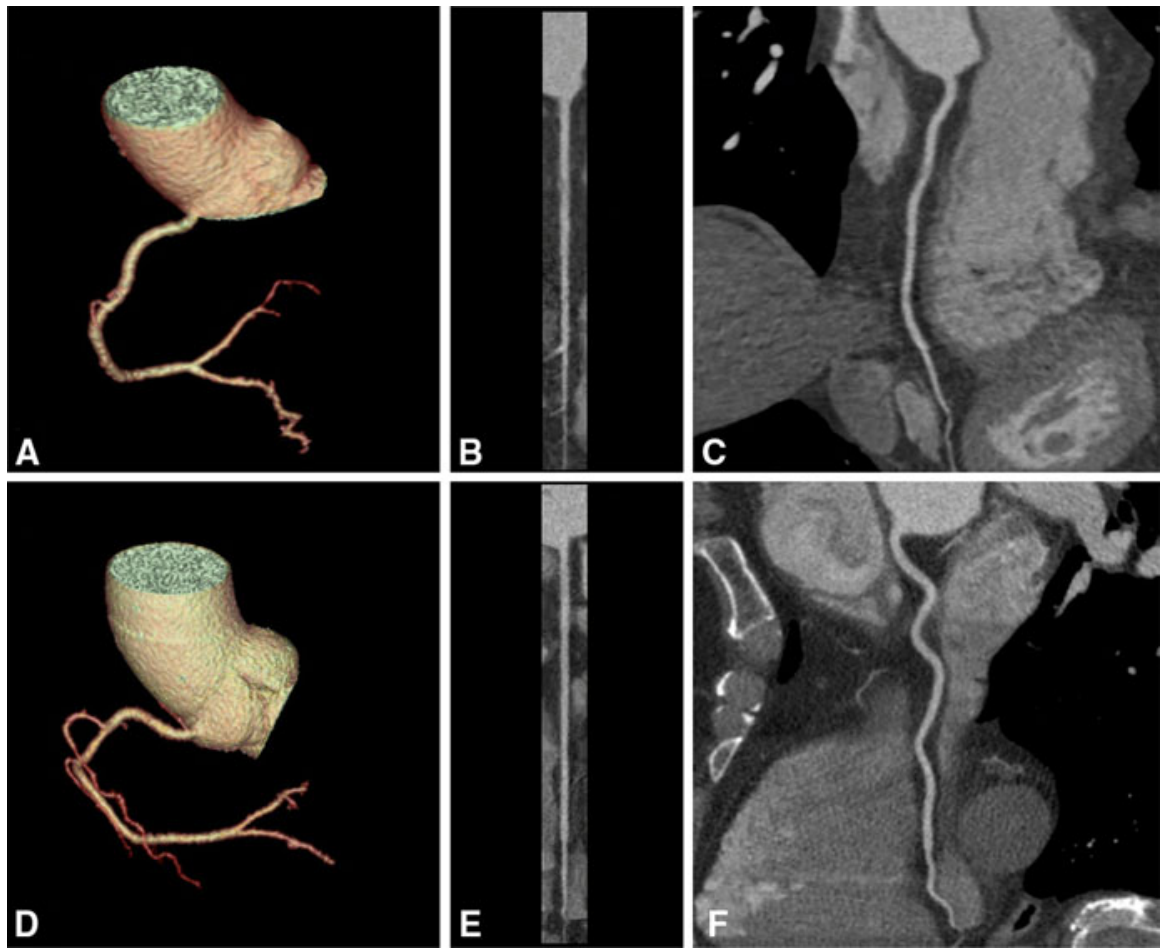

64-sequential scanning with 64-MSCT. In addition $\mathrm{X}$-rays irradiating the entire field of view after turning on the beam before starting the scan, expose the patient only once at the begin and at the end of the high-pitch spiral scan. By contrast, with prospective sequential scanning the unnecessary exposure occurs repeatedly for every sequential block.

Studies comparing radiation doses with 128-DSCT have reported even higher dose reduction rates than in the present study, however those comparisons were done against retrospective gating protocols and not versus prospective triggering mode [23]. The image quality which has also been assessed in the study by Duarte et al. [23] evaluated with different scanners 128-DSCT versus 64-MSCT using different scanning modes (high-pitch vs. retrospective scanning) has demonstrated superiority of the prospectively triggered high-pitch mode. This may be at, least in part, due to the fact that sequential scanning can induce stair-step artefacts due to irregular heart beat and can limit the accuracy of CCTA, this does not occur in spiral mode. In fact, in the present study stair step artefacts were observed in $18 \%$ of 64 -MSCT scans, but not in 128-DSCT scans.

Interestingly, while we found a significant correlation $(r=0.68, P \leq 0.01)$ between heart rate and image quality for 64-MSCT, in line with previous results [3, 24], no such correlation $(r=0.11$, $P=0.45$ ) was found for 128-DSCT. This is probably due to the fact that once heart rate is as low as to ensure a diastolic phase long enough to allow coverage of the whole coronary tree within one heart beat according to the prospective high-pitch spiral protocol, the absolute heart rate has no influence on image quality. By contrast, the prospectively triggered sequential scanning protocol involves several heart beats and requiring very low heart rate variability to avoid stair step artefacts [3, 24]. As increasing heart rates are more likely to be associated with heart rate variability image quality may be affected by higher heart rates explaining the correlation of heart rate and image quality score in 64-MSCT. However, higher heart rates over $70 \mathrm{bpm}$ may have more deleterious impact on image quality in 128-DSCT from 64-MSCT, but heart rates beyond $70 \mathrm{bpm}$ were excluded in the present study.

\section{Study limitations}

The image quality was visually assessed by a 4-pointscale and not by an automated system; this however, has been validated and documented to produce 
reliable results [3]. The present study does not represent a head-to-head comparison, as two different patient groups had to be identified because repeat scanning of the same patients would not be appropriate due to radiation exposure and ethical issues. However, we took due care to ascertain an optimal match of the two study groups with regard to the parameters identified as most relevant determinants of image quality [3, 25] in CCTA. All images were post-processed on the same workstation/software from one vendor to minimize the potential bias of different workstations/softwares on image quality, as it has been recently shown that differences in reconstruction algorithm may introduce more variability than different scanners. Finally, slice thickness was not equal in 128-DSCT versus 64-MSCT, which may affect image noise and consequently image quality. However, our study did not reveal any difference in noise from 128-DSCT versus 64-MSCT, excluding a relevant bias due to this technical issue.

\section{Conclusion}

128-DSCT with high-pitch spiral mode allows CCTA acquisition with reduced radiation dose at maintained image quality compared to 64-MSCT.

Acknowledgments We are grateful to Ennio Mueller, Edlira Loga, Mirjam De Bloeme, Sabrina Epp, and Daisy Beutel for their excellent technical support. The study was supported by a grant of the Swiss National Science Foundation (SNSF) and by the ZHIP (Zurich Center for Integrative Human Physiology, University of Zurich, Switzerland).

Conflict of interest None.

\section{References}

1. Hausleiter J, Meyer T, Hermann F et al (2009) Estimated radiation dose associated with cardiac $\mathrm{CT}$ angiography. JAMA 301:500-507

2. Husmann L, Valenta I, Gaemperli O et al (2008) Feasibility of low-dose coronary CT angiography: first experience with prospective ECG-gating. Eur Heart J 29:191-197

3. Herzog BA, Husmann L, Burkhard N et al (2009) Low-dose CT coronary angiography using prospective ECG-triggering: impact of mean heart rate and heart rate variability on image quality. Acad Radiol 16:15-21

4. Buechel RR, Husmann L, Herzog BA et al (2011) Lowdose computed tomography coronary angiography with prospective electrocardiogram triggering: feasibility in a large population. J Am Coll Cardiol 57:332-336

5. Kaufmann PA (2008) Low-dose computed tomography coronary angiography with prospective triggering: a promise for the future. J Am Coll Cardiol 52:1456-1457

6. Dewey M, Zimmermann E, Deissenrieder F et al (2009) Noninvasive coronary angiography by 320 -row computed tomography with lower radiation exposure and maintained diagnostic accuracy: comparison of results with cardiac catheterization in a head-to-head pilot investigation. Circulation 120:867-875

7. Alkadhi H, Stolzmann P, Desbiolles L et al (2010) Lowdose, 128-slice, dual-source CT coronary angiography: accuracy and radiation dose of the high-pitch and the stepand-shoot mode. Heart 96:933-938

8. Leschka S, Stolzmann P, Desbiolles L et al (2009) Diagnostic accuracy of high-pitch dual-source CT for the assessment of coronary stenoses: first experience. Eur Radiol 19:2896-2903

9. Hausleiter J, Bischoff B, Hein F et al (2009) Feasibility of dual-source cardiac CT angiography with high-pitch scan protocols. J Cardiovasc Comput Tomogr 3:236-242

10. Herzog BA, Husmann L, Burkhard N et al (2008) Accuracy of low-dose computed tomography coronary angiography using prospective electrocardiogram-triggering: first clinical experience. Eur Heart J 29:3037-3042

11. Goetti R, Feuchtner G, Stolzmann P et al (2010) Highpitch dual-source CT coronary angiography: systolic data acquisition at high heart rates. Eur Radiol 20:25652571

12. Herzog BA, Wyss CA, Husmann L et al (2009) First headto-head comparison of effective radiation dose from lowdose 64-slice CT with prospective ECG-triggering versus invasive coronary angiography. Heart 95:1656-1661

13. Pazhenkottil AP, Husmann L, Buechel RR et al (2010) Validation of a new contrast material protocol adapted to body surface area for optimized low-dose CT coronary angiography with prospective ECG-triggering. Int J Cardiovasc Imag 26:591-597

14. Husmann L, Herzog BA, Burkhard N et al (2009) Lowdose coronary CT angiography with prospective ECG triggering: validation of a contrast material protocol adapted to body mass index. Am J Roentgenol 193:802806

15. Austen WG, Edwards JE, Frye RL et al (1975) A reporting system on patients evaluated for coronary artery disease. Report of the Ad Hoc committee for grading of coronary artery disease, council on cardiovascular surgery, American heart association. Circulation 51:5-40

16. Goetti R, Leschka S, Baumuller S et al (2010) Low dose high-pitch spiral acquisition 128-slice dual-source computed tomography for the evaluation of coronary artery bypass graft patency. Investig Radiol 45:324-330

17. Husmann L, Herzog BA, Burger IA et al (2010) Usefulness of additional coronary calcium scoring in low-dose CT coronary angiography with prospective ECG-triggering impact on total effective radiation dose and diagnostic accuracy. Acad Radiol 17:201-206

18. Einstein AJ, Moser KW, Thompson RC et al (2007) Radiation dose to patients from cardiac diagnostic imaging. Circulation 116:1290-1305 
19. Einstein AJ (2009) Radiation protection of patients undergoing cardiac computed tomographic angiography. JAMA 301:545-547

20. Achenbach S, Marwan M, Ropers D et al (2010) Coronary computed tomography angiography with a consistent dose below $1 \mathrm{mSv}$ using prospectively electrocardiogram-triggered high-pitch spiral acquisition. Eur Heart J 31:340-346

21. Kaufmann PA, Knuuti J (2011) Ionizing radiation risks of cardiac imaging: estimates of the immeasurable. Eur Heart J 32:269-271

22. Husmann L, Herzog BA, Gaemperli O et al (2009) Diagnostic accuracy of computed tomography coronary angiography and evaluation of stress-only single-photon emission computed tomography/computed tomography hybrid imaging: comparison of prospective electrocardiogram-triggering versus retrospective gating. Eur Heart J 30:600-607
23. Duarte R, Bettencourt N, Costa JC et al (2010) Coronary computed tomography angiography in a single cardiac cycle with a mean radiation dose of approximately $1 \mathrm{mSv}$ : initial experience. Rev Port Cardiol 29:1667-1676

24. Husmann L, Herzog BA, Burkhard N et al (2009) Body physique and heart rate variability determine the occurrence of stair-step artefacts in 64-slice CT coronary angiography with prospective ECG-triggering. Eur Radiol 19:1698-1703

25. Tatsugami F, Husmann L, Herzog BA et al (2009) Evaluation of a body mass index-adapted protocol for low-dose 64-MDCT coronary angiography with prospective ECG triggering. AJR Am J Roentgenol 192:635-638 